\title{
Compromiso organizacional y sentimiento de permanencia de los profesionales en las organizaciones públicas y privadas de Lima Metropolitana
}

\author{
Organizational commitment and feeling of permanence of professionals in public \\ and private organizations in Lima \\ Alejandro Loli P.1', Willian Montgómery U., Fernando Cerón V., Javier del Carpio G., Vladimir Navarro V., Karen \\ LLACHO I. \\ Universidad Nacional Mayor de San Marcos, Lima, Perú
}

(RECIBIDO 10/03/2015, ACEPTADO 20/06/2015)

\begin{abstract}
RESUMEN
El estudio tiene por objeto conocer el compromiso organizacional y el sentimiento de permanencia de los profesionales en las organizaciones públicas y privadas de Lima. La muestra estuvo conformada por 221 empleados, la mayoría de ellos profesionales diversos que realizaban estudios de posgrado en centros de educación superior. Se les aplicó un cuestionario cuyos resultados nos indican que hay una tendencia favorable de compromiso organizacional, al igual que los sentimientos de permanencia en las entidades donde laboran. Por otro lado, se encontró que existe asociación significativa y positiva del compromiso afectivo con el sentimiento de permanencia, mientras que, este último, no se asocia con el compromiso de conveniencia. Entre tanto, la relación con las variables sociodemográficas exploradas es variada: el compromiso afectivo está relacionado con la edad, estado civil, antigüedad en el trabajo y tiempo de servicio en el puesto actual; el compromiso de conveniencia con la edad y el estado civil y el sentimiento de permanencia solo con la procedencia de los participantes; es decir, son variables diferenciadoras.
\end{abstract}

Palabras clave: Compromiso organizacional, sentimientos de permanencia, profesionales, Perú.

\begin{abstract}
The study aims to meet organizational commitment and feelings of permanence of professionals in public and private organizations in Lima. The sample consisted of 221 employees, most of them many professionals who are enrolled on graduate studies in higher education centers. A questionnaire was applied to them, the results indicate that there is a favorable trend of organizational commitment as feelings of stay in institutions where they work. On the other hand, we find that there is significant and positive association of affective commitment to the feeling of permanence, while the latter is not associated with the commitment
\end{abstract}

1 Docente principal de la Facultad de Psicología de la UNMSM. E-Mail: alolip@unmsm.edu.pe 
of convenience. Meanwhile, the relationship with socio demographic variables explored is varied, affective commitment is related to age, marital status, seniority and length of service in current position; the commitment of convenience to age and marital status and the feeling of permanence only to the origin of the participants; in other words, they are differentiating variables.

Keywords: Organizational Commitment, feelings of permanence, professional, Peru.

\section{INTRODUCCIÓN}

La globalización, el desarrollo tecnológico y el crecimiento económico están conduciendo a las organizaciones a valorizar mejor su capital humano a fin de incrementar la producción y mejorar la productividad; para cuyo efecto, demandan de altos niveles de compromiso de sus trabajadores que les permitan armonizar con las exigencias de la sociedad, es decir, solo con su involucramiento, identificación, su permanencia en la organización, se lograrán los objetivos esperados tanto en las instituciones públicas como privadas.

Por otro lado, contradictoriamente, la legislación laboral peruana permite diversas formas de contrato de la mano de obra, aplicables tanto a organizaciones públicas como privadas, no existiendo estabilidad laboral; vale decir, contratos a plazo determinado e indeterminado y la tercerización (empresas que contratan personal para dar servicio a terceros) que generan alto desplazamiento o rotación de los empleados en el mercado laboral; casos en los que el involucramiento, la identificación, la confianza, el compromiso, la satisfacción, la intención de permanencia en la organización, entre otros, como predictores o generadores de alta productividad, pierden sentido debido a la inestabilidad y la inseguridad en la organización en la que laboran. Situación a la que se añade la duración de las jornadas de trabajo que, en la mayoría de las organizaciones, especialmente privadas, es más de 10 horas diarias.

Arias Galicia (2000), en una investigación desarrollada sobre compromiso personal hacia la organización y la intención de permanencia, en una muestra de 177 empleados de una empresa privada de beneficiencia de México, encontró que el “... factor más importante para lograr el compromiso e incrementar la intención de permanencia es el apoyo organizacional...”. De allí la preocupación por conocer el compromiso organizacional y el sentimiento de permanencia de los profesionales en una muestra variada de instituciones privadas como públicas de Lima Metropolitana; es decir, las condiciones en las que laboran los profesionales en Perú pueden generar compromiso y sentimientos de permanencia. De hecho, tomando en cuenta que la efectividad de los miembros de una organización y sus sentimientos de permanencia en ella dependen en gran medida del compromiso que tenga con su organización, en Perú las organizaciones pueden estar generando todo lo contrario, salvo que exista reconocimiento al esfuerzo desplegado por los profesionales. 
En las últimas décadas, el factor humano es percibido como el único factor de la producción que da real ventaja competitiva a las organizaciones; en la medida en que los demás factores son sustituibles, se puede conseguir en el mercado con las mismas condiciones tecnológicas y financieras de la competencia. De allí se afirma que una alta productividad solo es posible con un capital humano comprometido (Gibson, Ivancebich y Donnelly, 1994), logrado a base de las condiciones ofrecidas por las organizaciones (una cultura organizacional con valores, un clima bien conducido, planes de desarrollo humano permanentes y sostenidos, etc.) durante su vida laboral; de manera que el compromiso organizacional que se construya en cada uno de sus miembros esté alineado a los principios, los valores, la misión y objetivos de la organización (Janssen, 2004; Freund, 2005).

Muchos autores definen el compromiso organizacional como el "grado en que un empleado participa en su trabajo" (Allport, 1943), "creencia en las metas" (Porter, Steers, Mowday y Boulain, 1974), "estado psicológico" (Meyer y Allen, 1991, 1993), “apego afectivo” (Bayona, Goñi y Madorrán, 2000), "estado interno de la persona" (Lagomarsino, 2003), "vínculos que mantienen ... apegado a una empresa" (Arciniega, s/f), "deber moral o psicológico adquirido" (Arias Galicia, 2003), identificación del individuo con la organización que lo emplea (Robbins, 2009: 79; Díaz y Montalbán, 2004), "vínculo o lazo del individuo con la organización” (Matihieu y Zajac, 1990).

Los estudios desarrollados en Perú no son muchos, han sido trabajados sobre la base de la propuesta de Meyer y Allen (1991), quienes indicaron que dicha relación entre una persona y una organización presenta consecuencias respecto a la decisión para continuar en la organización o dejarla. Según estos autores existen tres componentes del compromiso: afectivo, referido a lazos emocionales que las personas forjan con la organización al satisfacer sus necesidades y expectativa; de continuidad o conveniencia, referido a darse cuenta respecto a la inversión en tiempo y esfuerzo que se perderían en caso de dejar la organización para encontrar otro empleo, en otros términos, “...la intención de permanecer aparece como un cálculo de costo beneficio" en la percepción de March y Simon (1977), y el compromiso normativo, referido a la creencia en la lealtad a la organización en correspondencia a ciertas prestaciones (Meyer y Allen, 1991), debido, entre otros, al proceso de socialización que experimentan los trabajadores al incorporarse a la misma (Baker, 1990), desarrollando fuertes sentimientos de obligación de permanecer en la empresa (March, 1997).

Siguiendo la propuesta antes señalada de Meyer y Allen (1991), Loli (2005) da cuenta de una investigación que trata de conocer el compromiso organizacional de los trabajadores no docentes de una universidad pública de Lima y su relación con algunas variables demográficas en una muestra de 205 personas de un total de 20 facultades. Los resultados arrojan una tendencia favorable de los trabajadores hacia el compromiso organizacional y el análisis de los factores indica que existe correlación muy significativa y positiva entre satisfacción general con el 
trabajo y compromiso con el trabajo, compromiso afectivo, jerarquía del puesto y sentimiento de permanencia; mientras que la relación entre el primero y el compromiso de conveniencia es muy significativa pero negativa. Igual ocurre entre el compromiso con el trabajo y compromiso de conveniencia. También encontramos asociación muy significativa y positiva entre el compromiso con el trabajo y el compromiso afectivo, sentimiento de permanencia y jerarquía del puesto. Entre tanto, las variables demográficas respecto al compromiso organizacional no arroja ninguna relación; pero un análisis separado por cada uno de los factores del compromiso organizacional permite encontrar relación importante con educación, antigüedad en la universidad, antigüedad en el puesto actual y puesto actual.

Entre tanto, Arias Galicia (2001) desarrolló una investigación para conocer los factores que pueden propiciar las organizaciones para aumentar el compromiso de los empleados y la intención de permanencia en la organización. Se aplicaron cuestionarios a 177 empleados de una institución privada de beneficencia en México, analizando los datos mediante un modelo con base en ecuaciones estructurales. Los resultados indican que el factor más importante para lograr el compromiso e incrementar la intención de permanencia es el apoyo organizacional; es decir, el hecho de que la organización brinde un soporte en caso de que los empleados necesiten ayuda ante emergencias, además que se les considere como personas.

Otras investigaciones realizadas en Perú por los mismos autores (Arias Galicia y otros, 2003; Loli, 2006 y 2007) demuestran que no existe relación entre los componentes del compromiso organizacional, pero sí correlacionan con otras variables como satisfacción en el trabajo, compromiso con el trabajo, sentimiento de permanencia, claridad del rol, entre otras.

Sin embargo, revisando otros estudios se encuentra que el componente afectivo es el único de los tres, propuesto por Meyer y Allen (1997), que se sostiene sólido, en la medida en que está asociado a un conjunto importante de variables; mientras que el compromiso de conveniencia por tener una connotación de apego material del empleado está sujeto a sentimientos de permanencia condicionada, y el compromiso normativo es una obligación del empleado de permanecer en la organización por todos los beneficios obtenidos (Blanco, M; Castro, 2011), confirmando sus hallazgos con base en el análisis de datos para conocer el nivel de compromiso en una muestra de mujeres madres y no madres, cajeras de un banco de la ciudad de La Paz, Bolivia, llegando a la conclusión que no existe diferencia significativa entre madres y no madres en relación con el compromiso organizacional. El compromiso organizacional que más destaca en la muestra estudiada es el compromiso normativo; es decir, se sienten obligadas a permanecer en la organización, probablemente, debido a los beneficios y políticas internas del banco.

Pons y Ramos (2010), entre tanto, al realizar una investigación sobre la relación entre compromiso, autonomía, estabilidad laboral, y cuatro tipos de prácticas de 
recursos humanos (información compartida, formación y desarrollo, sistemas remunerativo), en una muestra de 458 sujetos, procedentes de 16 empresas privadas de Valencia, España, llegan a la conclusión que la participación y la autonomía en el puesto son variables que mejor se asocian con el compromiso, siendo menor la relación con las demás variables; por tanto -concluyen los autores-, las organizaciones que deseen mejorar el compromiso de sus miembros deben desarrollar programas de estimulación sobre dichas variables, más allá de consideraciones extrínsecas y/o garantías de permanencia en la empresa.

Ríos, Téllez y Ferrer (2010) informan de una investigación desarrollada sobre la relación entre las variables de empowerment y compromiso organizacional. Se utiliza como referencia un modelo generado con base en la descripción del empowerment en cuatro dimensiones: significado, competencia, autodeterminación e impacto; y del compromiso organizacional en tres: compromiso continuo, normativo y afectivo. Se aplicó un cuestionario a una muestra de 147 participantes de seis pymes localizadas en el municipio de Celaya, Guanajuato, México. Las empresas pertenecen a los sectores de servicios, comercio y producción. Sus hallazgos demuestran que el empowerment es un predictor del compromiso organizacional; y al analizar cada una de las dimensiones, se observa que solo el compromiso afectivo tiene un impacto directo en las cuatro dimensiones planteadas.

Mañas y col. (2007) presentan un estudio consistente en analizar el papel del compromiso de los empleados en la administración pública y reflejar las repercusiones que provoca en la institución, donde incluyen analizar variables como la satisfacción laboral, la satisfacción por la vida y el bienestar psicológico como antecedentes del compromiso. Para conseguir este objetivo, se aplicó un cuestionario a una muestra de 697 trabajadores pertenecientes a la administración pública. Los resultados reflejan que la mayor influencia se produce en la satisfacción laboral, seguida de la satisfacción por la vida y bienestar psicológico. Más aún, resaltamos el impacto que tiene el trabajo dinámico en la decisión del empleado de comprometerse con la entidad.

De las investigaciones revisadas se desprende el deseo de conocer la relación entre los componentes del compromiso organizacional: afectivo, continuo o conveniencia y normativo o sentimiento de permanencia de los profesionales en las organizaciones públicas y privadas de Lima, Perú; si bien es cierto que el compromiso organizacional, desde el punto de vista de la propuesta de Meyer y Allen (1991), está conformado por el compromiso afectivo, de conveniencia y el normativo, estas dos últimas variables no tienen la consistencia del compromiso afectivo, sino una connotación emocional de permanencia o no de los empleados en la organización. Mientras en el compromiso afectivo hay un lazo emocional de identificación y pertenencia que une al empleado con la organización, en el compromiso de conveniencia dicha vinculación está condicionada al costo beneficio, por lo que el sentimiento de retiro o permanencia está latente y en el compromiso normativo desarrollan un fuerte sentimiento de permanencia, como 
una obligación moral en correspondencia a las prestaciones que recibe de la organización, por tanto, sujeto a la actuación de la organización.

\section{MÉTODO}

\section{Muestra}

La muestra estuvo conformada por empleados que en su mayoría estaban cursando estudios de posgrado en diversas universidades (doctorados, maestrías, segunda especialidad o diplomado) y trabajan en entidades públicas o privadas de Lima o tienen formación básica pero ocupan cargos importantes por la experiencia lograda en el trabajo; por consiguiente, de diversas profesiones y ocupaciones. Así, el $80.5 \%$ tiene educación superior, $16.3 \%$ formación técnica, $2.3 \%$ secundaria completa y el $0.9 \%$ educación básica.

Tabla 1: Composición de la muestra segun variables demográficas

\begin{tabular}{cccc}
\hline Grupo etáreo & SEXO & ESTADO CIVIL & EDUCACIÓN \\
\hline $18-3031-4041-5051+$ & M F & C. D. S. V & Pri Sec. Tec. Sup. \\
98792915 & 109112 & 7751372 & 2536178 \\
221 & 221 & 221 & 221 \\
\hline
\end{tabular}

\section{Instrumento}

Para efectos del estudio se utilizó el cuestionario adaptado sobre la base de la prueba preparada por Fernando Arias Galicia. El instrumento está compuesto por reactivos relacionados a compromiso afectivo, de conveniencia o continuidad y sentimiento de permanencia o normativo (Meyer y Allen, 1991); satisfacción general en el trabajo (Eisenberger, 1997), referido a respuestas afectivas dadas en torno a su centro de trabajo; claridad del rol (Brown y Leigh, 1996), referido a la claridad de las expectativas, así como a las normas de trabajo; compromiso con el trabajo (Lodahl y Kejner, 1965), referido al grado en el cual la persona ha acogido los valores sobre la bondad e importancia de sus labores. Sin embargo, para efectos del presente estudio se analizarán solo los componentes propuestos por Meyer y Allen.

\section{Validez de constructo}

Administrado el instrumento y sometido a análisis (Prueba de KMO y Bartlett) permitió confirmar los factores teóricos existentes, lo que lleva a suponer que la prueba reúne las condiciones de validez de constructo estadísticamente significativo para el análisis factorial.

Tabla 2: Prueba de esfericidad

\begin{tabular}{lcc}
\hline & Prueba de KMO y Bartlett \\
\hline Medida Kaiser-Meyer-Olkin de adecuación de muestreo & .805 \\
& Aprox. Chi-cuadrado & 381.443 \\
Prueba de esfericidad de Bartlett & gl & 15 \\
& Sig. & .000 \\
\hline
\end{tabular}


Sin embargo, visto el número insuficiente de sujetos de la muestra $(\mathrm{m}=221)$ se procedió a realizar un análisis de componentes que arrojó que existen solo dos factores: el primero incluye el compromiso por conveniencia, la satisfacción general con el trabajo, la jerarquía del puesto, el compromiso afectivo y compromiso con el trabajo, mientras que el segundo factor solo lo constituye el sentimiento de permanencia, como se muestra en el siguiente cuadro. Esto significa que, para esta muestra, el sentimiento de permanencia es un factor relativamente independiente de los demás factores.

Tabla 3: Análisis de componentes principales

\begin{tabular}{ccc}
\hline & Matriz de patrón & \\
& & \multicolumn{3}{c}{ Componente } \\
\cline { 2 - 3 } & \multicolumn{1}{c}{1} & 2 \\
\hline Compromiso por conveniencia & .871 & \\
Compromiso afectivo & .742 & .969 \\
Sentimiento de permanencia & & \\
\hline
\end{tabular}

Método de extracción: análisis de componentes principales.

Método de rotación: Promax con normalización Kaiser. ${ }^{\text {a }}$

La rotación ha convergido en 3 iteraciones.

\section{Fiabilidad de la escala}

Como se puede apreciar en el siguiente cuadro, el coeficiente alpha de Crombach nos muestra que todos los factores cumplen con el criterio de estabilidad y confiabilidad, haciendo que el cuestionario total arroje un coeficiente $\mu=0.95$.

Tabla 4: Confiabilidad total por factores

\begin{tabular}{cc}
\hline Componentes & Alfa de Cronbach \\
\hline Compromiso de conveniencia & .84 \\
Compromiso afectivo & .86 \\
Sentimiento de permanencia & .34 \\
Confiabilidad total & .95 \\
\hline
\end{tabular}

Además de los ítems que forman parte del cuestionario, se incluyeron datos sociodemográficos como sexo, edad, grado de estudios, estado civil, tiempo de servicios en la organización, antigüedad en el puesto, entre otros.

\section{Procedimiento}

El cuestionario fue administrado en forma colectiva en salones de clase, a empleados que realizaban estudios de doctorado, maestría, segunda especialidad, diplomado o cursos libres en centros de enseñanza universitaria. 


\section{Procesamiento de datos}

El tratamiento estadístico se realizó haciendo uso del paquete estadístico SPSS (Statistical Package for the Social Sciences); entre ellos, se utilizó el estadístico de correlación de Spearman para establecer la asociación entre los diferentes factores. Fueron también importantes las medidas de tendencia central, la prueba Chi-cuadrado para muestras independientes aplicada a las variables sociodemográficas de dos grupos.

\section{RESULTADOS}

\section{Grado de compromiso organizacional}

La tabla 5 muestra el grado de compromiso organizacional de los empleados de organizaciones públicas y privadas de Lima, a través de tres factores (compromiso de conveniencia, compromiso afectivo y sentimiento de permanencia), y cuya tendencia es favorable hacia el compromiso organizacional. Esto significa que existe un alto porcentaje (25\%) de empleados que no se comprometen a plenitud con la organización dentro de los factores considerados.

Tabla 5: Grado de compromiso organizacional

\begin{tabular}{|c|c|c|c|c|c|}
\hline \multicolumn{6}{|c|}{ Nivel de compromiso } \\
\hline & & Frecuencia & Porcentaje & $\begin{array}{l}\text { Porcentaje } \\
\text { válido }\end{array}$ & $\begin{array}{l}\text { Porcentaje } \\
\text { acumulado }\end{array}$ \\
\hline \multirow{5}{*}{ Válido } & Desfavorable $<=\mathrm{Q} 1$ & 55 & 24.9 & 24.9 & 24.9 \\
\hline & Más o menos $>\mathrm{Q} 1 \mathrm{y}<=\mathrm{Q} 3$ & 111 & 50.2 & 50.2 & 75.1 \\
\hline & & & & & \\
\hline & Favorable $>$ Q3 & 55 & 24.9 & 24.9 & 100.0 \\
\hline & Total & 21 & 100.0 & 100.0 & \\
\hline
\end{tabular}

\section{Sentimiento de permanencia organizacional}

La tabla 6 muestra el sentimiento de permanencia organizacional de los empleados de organizaciones públicas y privadas de Lima, donde se puede apreciar que el $49 \%$ de ellos tiene la intención normal de permanecer dentro de su organización, el 22\% tiene una fuerte intención de permanecer y el $29 \%$ tiene una baja intención de permanecer en su institución. De lo que se puede deducir que un tercio de los empleados no están seguros de permanecer en la organización en la que trabajan. 
Tabla 6: Sentimiento de permanencia organizacional

\begin{tabular}{|c|c|c|c|c|c|}
\hline \multicolumn{6}{|c|}{ Nivel de permanencia } \\
\hline & & Frecuencia & Porcentaje & Porcentaje válido & $\begin{array}{l}\text { Porcentaje } \\
\text { acumulado }\end{array}$ \\
\hline \multirow{4}{*}{ Válido } & Desfavorable $<=$ Q1 & 65 & 29.4 & 29.4 & 29.4 \\
\hline & Más o menos $>$ Q1 y $<=$ Q3 & 108 & 48.9 & 48.9 & 78.3 \\
\hline & Favorable $>$ Q3 & 48 & 21.7 & 21.7 & 100.0 \\
\hline & Total & 221 & 100.0 & 100.0 & \\
\hline
\end{tabular}

\section{Relación entre el compromiso de conveniencia, el compromiso afectivo y el sentimiento de permanencia}

La tabla 7 muestra la correlación de los factores del compromiso organizacional de los empleados, donde se encontró que existe correlación muy significativa y positiva entre compromiso de conveniencia y compromiso afectivo ( $\mathrm{r}$ $\left.=.489^{* *}\right)$, y este último con el sentimiento de permanencia $\left(\mathrm{r}=.151^{*}\right)$; es decir, a mayor compromiso de conveniencia, mayor compromiso afectivo. Mientras que el compromiso de conveniencia no se asocia con el sentimiento de permanencia, lo que hace pensar que son independientes; probablemente debido a que el sentimiento de permanencia se basa en las prestaciones (empleo, salud, estabilidad, pensión y otros) de las organizaciones a sus empleados y estas son percibidas como insuficientes para que estos desarrollen dichos sentimientos.

Tabla 7: Correlación entre el compromiso de conveniencia y compromiso afectivo con el sentimiento de permanencia

\begin{tabular}{cccc|cc}
\hline Factores & Rho de Spearman & CC & \multicolumn{2}{c}{ CA } & \multicolumn{2}{c}{ SP } \\
\hline \multirow{2}{*}{ Compromiso de conveniencia } & Coeficiente de correlación & 1.000 & $.489^{* *}$ & -.002 \\
& Sig. (bilateral) &. & .000 & .979 \\
& $\mathrm{~N}$ & 221 & 221 & 221 \\
\hline \multirow{2}{*}{ Compromiso afectivo } & Coeficiente de correlación & $.489^{* *}$ & 1.000 & $.151^{*}$ \\
& Sig. (bilateral) &., 000 &. & .025 \\
\hline \multirow{2}{*}{ Sentimiento de permanencia } & $\mathrm{N}$ & 221 & 221 & 221 \\
& Coeficiente de correlación & -.002 & $.151^{*}$ & 1.000 \\
& Sig. (bilateral) & .979 & .025 &. \\
\hline
\end{tabular}

$\begin{array}{ll}(* *) \text { Muy significativo } & .01 \\ (*) \text { Significativo } & .05 \\ \mathrm{~N}=221 & \end{array}$

La correlación de la tabla 8 se confirma al realizar el análisis de componentes a partir de la validez de constructo, donde aparecen solo dos componentes: 1, conformado por el compromiso de conveniencia y compromiso afectivo; y el 2, conformado solo por sentimiento de permanencia, y la correlación es de .23, lo que significa que es positiva y baja. Por tanto, hay una relativa independencia entre los aspectos evaluados por la prueba. 
Tabla 8: Matriz de correlaciones de componente

\begin{tabular}{ccc}
\hline Componente & 1 & 2 \\
\hline 1 & 1.000 & .225 \\
2 & .225 & 1.000 \\
\hline
\end{tabular}

Método de extracción: análisis de componentes principales.

Método de rotación: Promax con normalización Kaiser.

Compromiso de conveniencia, compromiso afectivo y sentimiento de permanencia según variables sociodemográficas y organizacionales

Compromiso de conveniencia, compromiso afectivo y sentimiento de permanencia según edad

En la tabla 9, apreciamos el análisis comparativo según grupos etáreos, respecto a compromiso de conveniencia, compromiso afectivo y sentimiento de permanencia. A nivel descriptivo, respecto del compromiso de conveniencia se puede decir que participaron 221 empleados, de los cuales 98 sujetos tenían entre 18 y 30 años y un rango promedio de $98.85 ; 79$ sujetos tenían entre 31 y 40 años y su rango promedio fue de $116.14 ; 29$ sujetos tenían entre 41 y 50 años, con un promedio de 116.07; y 15 sujetos tenían entre 50 y 65 años y un rango promedio de 153.53.

Respecto del compromiso afectivo, participó el mismo número de muestra, del cual 98 sujetos tenían entre 18 y 30 años, quienes obtuvieron un rango promedio de $96.99 ; 79$ sujetos se encontraban entre 31 y 40 años y obtuvieron un rango promedio de 114.16; 29 sujetos entre 41 y 50 años obtuvieron 120.91 y 15 sujetos entre 50 años y más puntuaron 166.67. Entre tanto, respecto del sentimiento de permanencia, los promedios obtenidos por los sujetos fueron parejos; la diferencia entre los grupos etáreos no fue elevada.

Para establecer cuán significativas eran las diferencias, los datos se sometieron a la prueba de Chi-cuadrado, que nos permitió visualizar que las diferencias fueron positivas y significativas entre los grupos de edad tanto en compromiso de conveniencia ( $p$-value $<0.012$ ) como en compromiso afectivo ( $p$-value $<0.001$ ); lo que significa que la edad en estas dos variables juega un papel importante como elemento diferenciador, e, incluso, a mayor edad las puntuaciones son cada vez mayores. Entre tanto, respecto del sentimiento de permanencia encontramos que la diferencia no es significativa, es independiente.La edad no es una variable diferenciadora; es decir, el comportamiento de los sujetos se mantiene inalterable en todas las edades. 
Tabla 9: Compromiso de conveniencia, compromiso afectivo y sentimiento de permanencia según edad

\begin{tabular}{|c|c|c|c|}
\hline \multicolumn{4}{|c|}{ Rangos } \\
\hline & Edad & $\mathrm{N}$ & Rango promedio \\
\hline \multirow{5}{*}{ Compromiso por conveniencia } & 18 - 30 años & 98 & 98.85 \\
\hline & 31 - 40 años & 79 & 116.14 \\
\hline & $41-50$ años & 29 & 116.07 \\
\hline & $51-65$ años & 15 & 153.53 \\
\hline & Total & 221 & \\
\hline \multirow{5}{*}{ Compromiso afectivo } & $18-30$ años & 98 & 96.99 \\
\hline & $31-40$ años & 79 & 114.16 \\
\hline & $41-50$ años & 29 & 120.91 \\
\hline & $51-65$ años & 15 & 166.67 \\
\hline & Total & 221 & \\
\hline \multirow{6}{*}{$\begin{array}{l}\text { Sentimiento de } \\
\text { permanencia }\end{array}$} & 18 - 30 años & 98 & 108.81 \\
\hline & $31-40$ años & 79 & 110.99 \\
\hline & $41-50$ años & 29 & 120.10 \\
\hline & $51-65$ años & 15 & 107.77 \\
\hline & Total & 221 & \\
\hline & $\begin{array}{l}\text { Compromiso por } \\
\text { conveniencia }\end{array}$ & Compromiso afectivo & $\begin{array}{l}\text { Sentimiento de } \\
\text { permanencia }\end{array}$ \\
\hline Chi-cuadrado & 10.876 & 16.971 & .743 \\
\hline gl & 3 & 3 & 3 \\
\hline Sig. asintótica & .012 & .001 & .863 \\
\hline
\end{tabular}

a. Prueba de Kruskal Wallis

$\mathrm{p} \leq .05$

$\mathrm{p} \leq .01$

$\mathrm{N}=221$

\section{Compromiso de conveniencia, compromiso afectivo y sentimiento de permanencia según estado civil}

En la tabla 10, apreciamos el análisis comparativo según estado civil, respecto a compromiso de conveniencia, compromiso afectivo y sentimiento de permanencia. A nivel descriptivo, respecto del compromiso de conveniencia se puede decir que participaron 221 empleados, de los cuales 77 sujetos eran casados, con un rango promedio de 124.66; 5 sujetos eran divorciados y su rango promedio fue de 139.40; 137 sujetos eran solteros, con un promedio de 101.24; y 2 sujetos eran viudos y un rango promedio de 182.75 .

Respecto del compromiso afectivo, participó el mismo número de muestra, del cual 77 sujetos eran casados, quienes obtuvieron un rango promedio de 126.82; 5 sujetos eran divorciados y obtuvieron un rango promedio de $127.40 ; 137$ sujetos eran solteros y obtuvieron 100.56 y 2 sujetos eran viudos y puntuaron 176.00. Entre tanto, respecto del sentimiento de permanencia, los promedios obtenidos por los sujetos no fueron muy distintos. 
Los datos, para establecer cuán significativas eran las diferencias, se sometieron a la prueba de Chi-cuadrado, que nos permitió apreciar que las diferencias por grupos de acuerdo al estado civil de los sujetos fueron positivas y significativas, tanto en compromiso de conveniencia $(\mathrm{p}$-value $<0.017$ ) como en compromiso afectivo (p-value $<0.013$ ); lo que significa que la condición del estado civil en estas dos variables juega un papel importante como elemento diferenciador. Entre tanto, respecto del sentimiento de permanencia encontramos que la diferencia no es significativa, es independiente, el estado civil no es una variable diferenciadora; es decir, el comportamiento de los sujetos no varía, se da de la misma manera cuando se trata del sentimiento de permanencia.

Tabla 10: Compromiso de conveniencia, compromiso afectivo y sentimiento de permanencia según estado civil

\begin{tabular}{cccc}
\hline & Rangos & & \\
\hline & Estado civil & $\mathrm{N}$ & Rango promedio \\
\hline \multirow{4}{*}{ Compromiso por conveniencia } & Casado & 77 & 124.66 \\
& Divorciado & 5 & 139.40 \\
& Soltero & 137 & 101.24 \\
& Viudo & 2 & 182.75 \\
& Total & 221 & \\
\hline \multirow{3}{*}{ Compromiso afectivo } & Casado & 77 & 126,82 \\
& Divorciado & 5 & 127.40 \\
& Soltero & 137 & 100.56 \\
& Viudo & 2 & 176.00 \\
& Total & 221 & \\
\hline \multirow{3}{*}{ Sentimiento de permanencia } & Casado & 77 & 89.00 \\
& Divorciado & 5 & 107.89 \\
& Soltero & 137 & 80.75 \\
& Viudo & 2 & \\
\hline \multirow{3}{*}{ Compromiso total } & Total & 221 & 123.77 \\
& Casado & 77 & 105.60 \\
& Divorciado & 5 & 103.69 \\
& Soltero & 137 & 133.50 \\
& Viudo & 2 & \\
\hline
\end{tabular}

\begin{tabular}{cccc}
\hline & $\begin{array}{c}\text { Compromiso por } \\
\text { conveniencia }\end{array}$ & Compromiso afectivo & $\begin{array}{c}\text { Sentimiento de } \\
\text { permanencia }\end{array}$ \\
\hline Chi-cuadrado & 10.219 & 10.772 & 2.499 \\
gl & 3 & 3 & 3 \\
Sig. asintótica & .017 & .013 & .475 \\
\hline
\end{tabular}

a. Prueba de Kruskal Wallis

$\mathrm{p} \leq .05$

$\mathrm{N}=221$ 


\section{Compromiso de conveniencia, compromiso afectivo y sentimiento de permanencia según procedencia}

La tabla 11 presenta el análisis comparativo según la procedencia de los sujetos respecto al compromiso de conveniencia, compromiso afectivo y sentimiento de permanencia. A nivel descriptivo, respecto del compromiso de conveniencia y el compromiso afectivo se puede decir que participaron 220 empleados, cuyos rangos promedios de los procedentes de Lima y provincias no son muy diferentes unos de otros.

Entre tanto, respecto del sentimiento de permanencia, con una muestra igual de sujetos, 150 procedían de Lima y obtuvieron un rango promedio de 117.36; 70 procedían del interior del país (provincias) y obtuvieron un rango promedio de 95.79; permitiendo deducir que la distancia entre los promedios es amplia.

Un análisis de los datos para establecer el grado de significancia, respecto del sentimiento de permanencia, nos llevó a someterlos al puntaje "Z", que nos permitió apreciar que las diferencias entre los grupos de sujetos por procedencia fueron positivas y significativas ( $\mathrm{p}$-value $<0.019$ ); lo que significa que el comportamiento de los participantes de la muestra que proceden de Lima es diferente de los procedentes de provincias y estas dos variables juegan un papel importante como elemento diferenciador, siendo mayor el promedio para los procedentes de provincias. Entre tanto, respecto del compromiso de conveniencia y afectivo, la diferencia no es significativa, la procedencia es independiente, no es una variable diferenciadora; el comportamiento de los sujetos es homogéneo.

Tabla 11: Compromiso de conveniencia, compromiso afectivo y sentimiento de permanencia según procedencia

\begin{tabular}{ccccc}
\hline \multicolumn{5}{c}{ Rangos } \\
Factores & Procedencia & $\mathrm{N}$ & Rango promedio & Suma de rangos \\
\hline \multirow{3}{*}{ Compromiso por conveniencia } & Lima & 150 & 106.82 & 16023.00 \\
& Provincias & 70 & 118.39 & 8287.00 \\
& Total & 220 & & \\
\hline \multirow{2}{*}{ Compromiso afectivo } & Lima & 150 & 112.41 & 16862.00 \\
& Provincias & 70 & 106.40 & 7448.00 \\
& Total & 220 & & \\
\hline \multirow{2}{*}{ Sentimiento de permanencia } & Lima & 150 & 117.36 & 17604.50 \\
& Provincias & 70 & 95.79 & 6705.50 \\
& Total & 220 & & \\
\hline
\end{tabular}

Prueba de Mann-Whitney

$\mathrm{N}=220$ 


\begin{tabular}{cccc}
\hline & $\begin{array}{c}\text { Compromiso por } \\
\text { conveniencia }\end{array}$ & $\begin{array}{c}\text { Compromiso } \\
\text { afectivo }\end{array}$ & $\begin{array}{c}\text { Sentimiento de } \\
\text { permanencia }\end{array}$ \\
\hline U de Mann-Whitney & 4698.000 & 4963.000 & 4220.500 \\
W de Wilcoxon & 16023.000 & 7448.000 & 6705.500 \\
Sig. asintótica (bilateral) & -1.256 & -.653 & -2.345 \\
\hline
\end{tabular}

a. Variable de agrupación: Procedencia

$\mathrm{p} \leq .05$

$\mathrm{N}=220$

Compromiso de conveniencia, compromiso afectivo y sentimiento de permanencia según años de antigüedad en la organización

La tabla 12 presenta el análisis comparativo según años de antigüedad de los sujetos respecto a compromiso de conveniencia, compromiso afectivo y sentimiento de permanencia. A nivel descriptivo, respecto del compromiso de conveniencia y el sentimiento de permanencia, participaron 221 empleados, cuyos rangos promedios de los grupos por años de antigüedad no son muy diferentes unos de otros.

Entre tanto, respecto del compromiso afectivo, con una muestra similar, 61 de ellos tenían hasta un año de antigüedad y obtuvieron un rango promedio de $85.63 ; 81$ sujetos tenían entre 2 y 5 años de antigüedad y tuvieron un rango promedio de 105.86; 46 participantes tenían entre 6 y 10 años y obtuvieron un rango promedio de 122.68 ; y 33 personas tenían más de 10 años y obtuvieron un rango promedio de 154.23 , lo que permitió observar que la distancia entre los promedios es amplia.

El análisis de los datos para establecer el grado de significancia respecto del compromiso afectivo permitió someterlos a la prueba Chi-cuadrado, que indica las diferencias entre los grupos de sujetos por años de antigüedad, siendo estas positivas y significativas ( $p$-value $<0.000$ ); lo que quiere decir que el comportamiento de los participantes de la muestra juega un papel importante como elemento diferenciador, siendo mayor el rango promedio a medida que pasan los años de trabajo en la organización. Entre tanto, respecto del compromiso de conveniencia y sentimiento de permanencia, la diferencia no es significativa; los años de trabajo en la organización como variable es independiente, no es una variable diferenciadora. 
Tabla 12: Compromiso de conveniencia, compromiso afectivo y sentimiento de permanencia según años de antigüedad en la organización

\begin{tabular}{|c|c|c|c|}
\hline \multicolumn{4}{|c|}{ Rangos } \\
\hline & Antigüedad en el servicio & $\mathrm{N}$ & Rango promedio \\
\hline \multirow{5}{*}{ Compromiso por conveniencia } & 1 año & 61 & 98.04 \\
\hline & 2 - 5 años & 81 & 110.37 \\
\hline & $6-10$ años & 46 & 114.71 \\
\hline & 10 años a más & 33 & 131.33 \\
\hline & Total & 221 & \\
\hline \multirow{5}{*}{ Compromiso afectivo } & 1 año & 61 & 85.63 \\
\hline & 2 - 5 años & 81 & 105.86 \\
\hline & $6-10$ años & 46 & 122.68 \\
\hline & 10 años a más & 33 & 154.23 \\
\hline & Total & 221 & \\
\hline \multirow{5}{*}{ Sentimiento de permanencia } & 1 año & 61 & 109.24 \\
\hline & 2 - 5 años & 81 & 110.56 \\
\hline & $6-10$ años & 46 & 112.39 \\
\hline & 10 años a más & 33 & 113.41 \\
\hline & Total & 221 & \\
\hline
\end{tabular}

Prueba de Kruskal-Wallis

$$
\begin{gathered}
\text { Compromiso por } \\
\text { conveniencia }
\end{gathered} \text { Compromiso afectivo } \quad \begin{gathered}
\text { Sentimiento de } \\
\text { permanencia }
\end{gathered}
$$

\begin{tabular}{cccc}
\hline Chi-cuadrado & 6.008 & 26.760 & .119 \\
gl & 3 & 3 & 3 \\
Sig. asintótica & .111 & .000 & .989 \\
\hline
\end{tabular}

a. Prueba de Kruskal Wallis

$\mathrm{p} \leq .01$

$\mathrm{N}=221$

Compromiso de conveniencia, compromiso afectivo y sentimiento de permanencia según los años de servicio en el puesto de trabajo

La tabla 13 presenta el análisis comparativo según el tiempo de servicio de los sujetos en el puesto de trabajo respecto al compromiso de conveniencia, compromiso afectivo y sentimiento de permanencia. A nivel descriptivo, respecto del compromiso de conveniencia y el sentimiento de permanencia, participaron 221 empleados, cuyos rangos promedios de los grupos por años de servicio en el puesto no son muy diferentes unos de otros. 
Entre tanto, respecto del compromiso afectivo, con una muestra similar, 81 de ellos tenían hasta un año de servicio en el puesto y obtuvieron un rango promedio de 96.29; 96 sujetos tenían entre 2 y 5 años de servicio y tuvieron un rango promedio de 109.11; 34 participantes tenían entre 6 y 10 años y obtuvieron un rango promedio de 138.63 ; y 10 personas tenían más de 10 años y obtuvieron un rango promedio de 154.35 , lo que permite observar que la distancia entre los promedios es amplia.

El análisis de los datos para establecer el grado de significancia, respecto del compromiso afectivo, permitió someterlos a la prueba Chi-cuadrado, que indica las diferencias entre los grupos de sujetos por tiempo de servicio en el puesto, siendo estas positivas y significativas ( -value $<0.002$ ); lo que significa que el comportamiento de los participantes de la muestra juega un papel importante como elemento diferenciador, siendo mayor el rango promedio a medida que pasan los años de servicio. Entre tanto, respecto del compromiso de conveniencia y sentimiento de permanencia, la diferencia no es significativa; los años de servicio en el puesto como variable es independiente, no es una variable diferenciadora.

Tabla 13: Compromiso de conveniencia, compromiso afectivo y sentimiento de permanencia según tiempo de servicio en el puesto de trabajo

\begin{tabular}{cccc}
\hline & Rangos & & \\
& Años en el puesto & $\mathrm{N}$ & Rango promedio \\
\hline \multirow{3}{*}{ Compromiso por conveniencia } & 1 año & 81 & 107.79 \\
& $2-5$ años & 96 & 109.87 \\
& $6-10$ años & 34 & 113.07 \\
& 10 años a más & 10 & 140.80 \\
& Total & 221 & \\
\hline \multirow{2}{*}{ Compromiso afectivo } & 1 año & 81 & 96.29 \\
& $2-5$ años & 96 & 109.11 \\
& $6-10$ años & 34 & 138.63 \\
& 10 años a más & 10 & 154.35 \\
& Total & 221 & \\
\hline \multirow{2}{*}{ Sentimiento de permanencia } & 1 año & 81 & 109.89 \\
& $2-5$ años & 96 & 114.13 \\
& $6-10$ años & 34 & 106.26 \\
& 10 años a más & 10 & 106.05 \\
\hline
\end{tabular}

Prueba de Kruskal-Wallis

\begin{tabular}{cccc}
\hline & $\begin{array}{c}\text { Compromiso por } \\
\text { conveniencia }\end{array}$ & Compromiso afectivo & $\begin{array}{c}\text { Sentimiento de } \\
\text { permanencia }\end{array}$ \\
\hline Chi-cuadrado & 2.443 & 15.326 & .503 \\
gl & 3 & 3 & 3 \\
Sig. asintótica & .486 & .002 & .918 \\
\hline
\end{tabular}

a. Prueba de Kruskal Wallis

b. Variable de agrupación: Años en el puesto

$\mathrm{p} \leq .05$

$\mathrm{N}=221$ 
Finalmente, las demás variables demográficas, tales como el sexo y lugar de residencia de los participantes en la muestra, no son significativas, no son variables diferenciadoras; por tanto, son homogéneas y no influyen esas condiciones sobre el compromiso de conveniencia, compromiso afectivo y sentimiento de pertenencia que pueden desarrollar los sujetos.

\section{DISCUSIÓN}

La preocupación por conocer la relación entre los componentes del compromiso organizacional -afectivo, continuo o conveniencia y normativo, propuesto por Meyer y Allen (1991)- de los profesionales en las organizaciones públicas y privadas de Lima, Perú, surge debido a la variabilidad de los resultados de diversos estudios sobre estas relaciones. De manera que se plantea como compromiso organizacional solo las dos primeras, distinguiéndolas del normativo, o denominado también como sentimiento de permanencia, por considerar que este componente es independiente del compromiso afectivo y del compromiso de conveniencia, como efectivamente lo demuestran los resultados del presente estudio. Así, el compromiso afectivo es el único componente que tiene impacto directo con los componentes del empoderamiento como predictor del compromiso organizacional (Ríos, Téllez y Ferrer, 2010), con la satisfacción en el trabajo, compromiso con el trabajo, jerarquía del puesto, sentimiento de permanencia (Loli, 2005), claridad del rol (Arias; Loli y otros, 2003), la participación y la autonomía (Pons y Ramos, 2010); este último decía que estas variables se asocian mejor con el compromiso, más allá de consideraciones extrínsecas y/o garantías de permanencia en la empresa.

Como se puede apreciar, los resultados señalados confirman nuestros hallazgos de que el compromiso afectivo está asociado al compromiso de conveniencia y más débilmente al compromiso normativo o sentimiento de permanencia, probablemente debido al lazo emocional de los empleados con la organización, pero el compromiso de conveniencia no se asocia con este último, lo que permite deducir que si bien es cierto que tanto el compromiso de conveniencia como el compromiso normativo tienen la connotación de permanecer o no en la organización, el primero está vinculado al costo beneficio que percibe el empleado si tuviera que retirarse (Meyer y Allen, 1991), mientras que el segundo está vinculado a la obligación moral; es decir, se sienten obligados a permanecer en la organización, probablemente, debido a los beneficios y políticas internas de organización (Blanco, Castro, 2011); ya decía Arias G. (2001) que el factor más importante para lograr el compromiso e incrementar la intención de permanencia es el apoyo organizacional; es decir, el hecho de que la organización brinde un soporte en caso de que los empleados necesiten ayuda ante emergencias, además de considerarlos como personas. En el mejor de los casos, puede deberse a la formación del valor de lealtad en el individuo desde su niñez, es decir, el sujeto aprende y da por hecho que debe ser leal con la empresa que lo contrate, desde luego la aplicación de este valor se hace presente en otros contextos de la vida de la persona (March, 1997), siempre que 
la organización responda a la altura de dicha lealtad. Aunque esta última posición parece ajustarse mejor al compromiso afectivo.

Finalmente, es pertinente señalar que el compromiso afectivo y de conveniencia de los participantes se afecta cuando intervienen las variables sociodemográficas como la edad y el estado civil. En cambio, esta variación se puede apreciar solo con el compromiso afectivo cuando se trata de antigüedad en la organización y en el puesto. Mientras, ocurre lo mismo con el sentimiento de permanencia, que varía el comportamiento de los participantes de acuerdo a la procedencia.

No obstante, se debe indicar que la investigación adolece de limitaciones en el número de muestra, que no fue suficiente para realizar el análisis factorial, aunque nos permitió conocer la independencia del compromiso normativo o sentimiento de permanencia respecto de los otros dos componentes señalados por Meyer y Allen (1991). Esta situación nos conduce a creer que requiere de mayor exploración y que no es todavía posible generalizar los resultados.

\section{CONCLUSIONES}

1. En términos generales, el grado de compromiso organizacional de los empleados de organizaciones públicas y privadas de Lima, a través de tres factores (compromiso de conveniencia, compromiso afectivo y normativo o sentimiento de permanencia) es favorable; sin embargo, existe un alto porcentaje (25\%) de empleados que no se compromete a plenitud con la organización dentro de los factores considerados.

2. Un análisis separado sobre el factor normativo o sentimiento de permanencia de los empleados de entidades públicas y privadas de Lima permite apreciar que hay una tendencia favorable de permanecer dentro de su organización; sin embargo, existe un tercio (29\%) de ellos que tiene un bajo sentimiento de permanencia en su institución.

3. Existe correlación muy significativa y positiva entre compromiso de conveniencia y compromiso afectivo; una asociación débil entre el compromiso afectivo y el sentimiento de permanencia; es decir, a mayor compromiso de conveniencia, mayor compromiso afectivo, y a mayor compromiso afectivo, mayor sentimiento de permanencia. Mientras, el compromiso de conveniencia no se asocia con el sentimiento de permanencia, lo que hace pensar que son independientes, situación que se confirma con el análisis de componentes.

4. El compromiso afectivo y de conveniencia de los participantes se afecta cuando intervienen las variables de edad y estado civil; en cambio, cuando intervienen las variables como antigüedad en la organización y en el puesto, influyen en el compromiso afectivo. Mientras, ocurre de manera similar con el sentimiento de permanencia, que varía el comportamiento de los participantes de acuerdo al lugar de donde proceden. 


\section{REFERENCIAS BIBLIOGRÁFICAS}

Allen, J. y Meyer, P. (1993). Organizational commitment: evidence of career stage effects? Journal of Business Research, 26: 49-61.

Arias Galicia, F. (2001). El compromiso personal hacia la organización y la intención de permanencia: algunos factores para su incremento. Rev. de Contaduría y Administración, No 200, enero marzo 2001. México, Edit. UNAM.

Arias, F.; Varela, D.; Loli, A.; y Quintana, M. (2003). El compromiso organizacional y su relación con algunos factores demográficos. LimaPerú: Rev. de Investigación en Psicología, vol. 6, Nº 2, UNMSM.

Baker, W. (1995). Allen and Meyer's 1990 Longitudinal Study: A Reanalysis and Reinterpretation Using Structural Equation Modeling. Human Relations, 48, 2, págs. 169-185.

Blanco, M. y Castro, P. (2011). Ajayu, 9(2). Análisis comparativo del compromiso organizacional manifestado por madres y mujeres sin hijos. La Paz, Bolivia: Universidad Católica Boliviana "San Pablo".

Díaz Bretones, F. y Montalbán Peregrín, M. (2004). El individuo como unidad de análisis: la influencia de los valores, percepciones y actitudes en el comportamiento laboral (en A. Rodríguez: coord.), Psicología de las Organizaciones. Barcelona: Editorial UOC. pp. 125-166.

Mañas, M; Salvador, C; Boada, J; González, E. y Agulló, E. (2007). La satisfacción y el bienestar psicológico como antecedentes del compromiso organizacional. Oviedo, España:Psicothema, Vol. 19, nº 3, pp. 395-400.

Mathieu, E. y Zajac, M. (1990). A review and meta-analysis of the antecedents, correlates and consequences of organizational commitment. Psychological Bulletin, 180, 171-194.

March, J. H. Simón (1977). Teoría de la organización. Barcelona: Ariel (Links)

Loli Pineda, Alejandro (2005). Compromiso organizacional de los trabajadores de una universidad pública de Lima y su relación con algunas variables demográficas. Instituto de Investigaciones Psicológicas, Lima, Perú: UNMSM.

Pons V. F y Ramos L. J. (2010). Antecedentes del compromiso organizacional: Relaciones con la estabilidad laboral, la autonomía en el puesto y las prácticas de recursos humanos. Rev. Trabajo y Seguridad Social, CEF, 323, pp. 239-256. Universidad de Valencia, España.

Ríos M.*, Téllez M. y Ferrer J. (2010). El empowerment como predictor del compromiso organizacional en las Pymes. México: UNAM, Facultad de Contaduría y Administración. 